\section{Endoscopic argon plasma coagulation for the treatment of hemorrhagic pseudopolyps in colonic Crohn's disease}
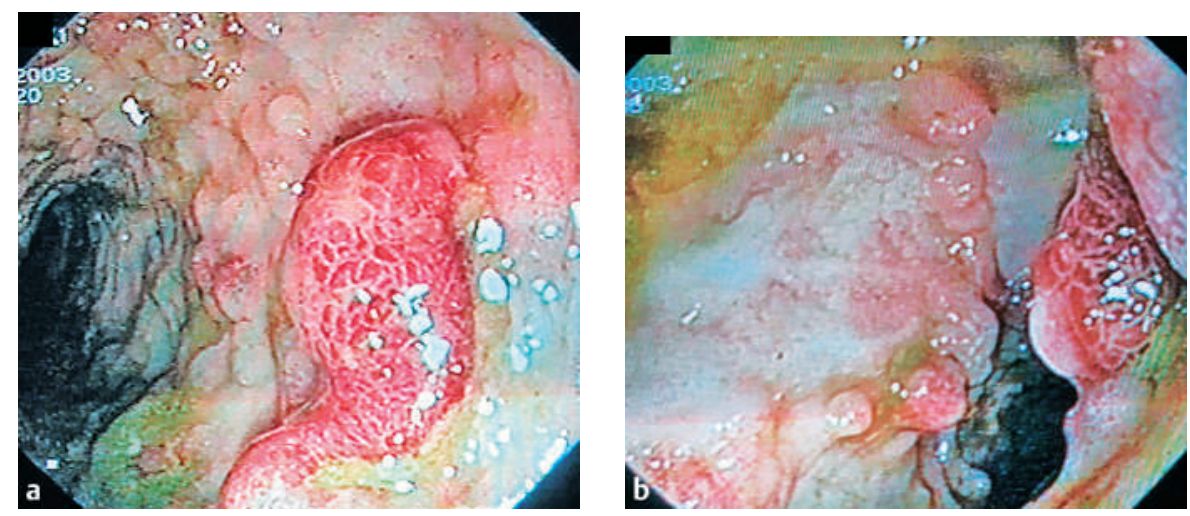

Figure 1 Two colonoscopic views of the hemorrhagic pseudopolyps seen at the first colonoscopy. The mucosa has an erythematous and mosaic appearance, caused by edema and vascular congestion.

A 32-year-old woman with a history of colonic Crohn's disease since 1997, who had been on azathioprine since 2002, presented in April 2003 with sudden-onset hematochezia and a drop in hemoglobin to $6.2 \mathrm{~g} / \mathrm{dL}$, but without a clinical flare of her Crohn's disease. Colonoscopy showed multiple pseudopolyps from the rectum to the splenic flexure, with fresh blood in the colonic lumen. Some of these pseudopolyps had an erythematous and mosaic appearance, but there was no ulceration (Figure 1). No signs of Crohn's colitis or active bleeding were observed. Severe bleeding continued on a daily basis and necessitated treatment with 2 units of blood per day to maintain a hemoglobin level higher than $8 \mathrm{~g} / \mathrm{dL}$. A colectomy was considered.

At day 5, a second colonoscopy showed an oozing of blood welling up from a small hemorrhagic pseudopolyp, which confirmed that these lesions were causing the hemorrhage (Figure 2). As a trial, the bleeding zone and all the erythematous pseudopolyps were treated by argon plasma coagulation (50 watts, $1.5 \mathrm{~L} /$ minute). This dramatically reduced the hematochezia in 4 days. Surgery was cancelled and the hemoglobin remained stable at $11 \mathrm{~g} / \mathrm{dL}$. Hematochezia recurred at day 20 and to a lesser degree at day 40 , with recorded hemoglobin levels of $7 \mathrm{~g} / \mathrm{dL}$ and $9 \mathrm{~g} / \mathrm{dL}$ respectively. Argon plasma coagulation was performed each time, focused specifically on the most erythematous pseudopolyps. No other bleeding occurred, and at 2 months her hemoglobin was stable at $12 \mathrm{~g} / \mathrm{dL}$. It was decided that control colonoscopy would not be useful because of the successful control of bleeding and the fact that her Crohn's disease was not active. She was maintained on azathioprine therapy and follow-up with regular monitoring by the referring physician (A.A.) showed that she experienced no recurrence of the bleeding or flare of her disease over the next 3 years.

Pseudopolyps are an exceptional cause of bleeding in Crohn's disease and surgical treatment is required in most cases $[1,2]$. This case illustrates the first report of the use of argon plasma coagulation in Crohn's disease, representing a new potential indication for this treatment modality.

Endoscopy_UCTN_Code_CCL_1AD_2AD

Endoscopy_UCTN_Code_TTT_1AQ_2AZ
A. Attar', C. Bon ${ }^{2}$, V. Sebbagh ${ }^{3}$, B. Béjou ${ }^{2}$, R. Bénamouzig ${ }^{2}$
${ }^{1}$ Service de Gastroentérologie et Assist- ance Nutritive, Hôpital Beaujon, Clichy, France
${ }^{2}$ Service d'Hépato-Gastroentérologie, Hôpital Avicenne, Bobigny, France
${ }^{3}$ Service d'Hépato-Gastroentérologie, Hôpital Saint-Antoine, Paris, France.

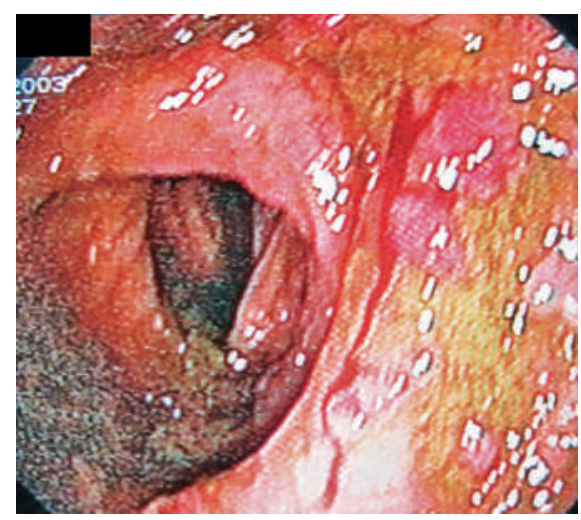

Figure 2 Active oozing bleeding from a small hemorrhagic pseudopolyp was seen at the second colonoscopy.

\section{References}

${ }^{1}$ Pardi D, Loftus E, Tremaine $\mathrm{W}$ et al. Acute major gastrointestinal hemorrhage in inflammatory bowel disease. Gastrointest Endosc 1999; 49: 153-157

2 Belaiche J, Louis E, D'Haens G et al. Acute lower gastrointestinal bleeding in Crohn's disease: characteristics of a unique series of 34 patients. Belgian IBD Research Group. Am J Gastroenterol 1999; 94: 2177-2181

\section{Corresponding author}

\section{A. Attar, MD}

Hôpital Beaujon

100 Boulevard du Général Leclerc

92110 Clichy

France

Fax: +33-1-40-87-45-74

Email: alain.attar@bjn.aphp.fr 